\title{
La resolución de problemas en los libros de texto: un instrumento para su análisis
}

\author{
Elena María López, Universidad de Huelva (España) \\ Ana Cristina Guerrero, IES Licinio de la Fuente (España) \\ José Carrillo, Universidad de Huelva (España) \\ Luis Carlos Contreras, Universidad de Huelva (España)
}

Recibido el 23 de Abril de 2015; aceptado el 13 de Octubre de 2015

\section{La resolución de problemas en los libros de texto: un instrumento para su análisis}

\section{Resumen}

Este artículo muestra un instrumento para analizar el papel de la resolución de problemas en los libros de texto. Partimos de la descripción del papel otorgado a los libros de texto en los procesos de enseñanza y aprendizaje de las matemáticas, para pasar después a mostrar algunas investigaciones que, desde la educación matemática, se han realizado sobre libros de texto, entre ellas las llevadas a cabo en nuestro grupo. De estas últimas mostraremos ejemplos de cómo el instrumento de análisis señalado permite analizar los tipos de problemas que aparecen en los libros de texto.

Palabras clave. Resolución de problemas; libros de texto de matemática; instrumento de análisis.

\section{A resolução de problemas em livros didáticos: um instrumento para análise}

\section{Resumo}

Este artigo mostra um instrumento para analisar o papel da resolução de problemas em livros didáticos. Começamos com a descrição do papel dado aos livros didáticos no ensino e aprendizagem da matemática, em seguida, passar para mostrar alguma pesquisa da educação matemática, têm sido realizados sobre livros didáticos, incluindo os realizados colocar em nosso grupo. Destes exemplos mostram como a ferramenta de análise apontou para analisar os tipos de problemas que aparecem nos livros didáticos.

Palavras chave. Solução de problemas; livros didáticos de matemática; ferramenta de análise.

\section{Problem solving in textbooks: an instrument for analysis}

\section{Abstract}

This article shows an instrument to analyse the role of problem solving in textbooks. We begin with the description of the role given to the textbooks in the teaching and learning of mathematics, then move on to show some research on textbooks from mathematics education, including those carried out in our

Para citar: López, E.M., Guerrero, A.C., Carrillo, J. \& Contreras, L.C. (2015). La resolución de problemas en los libros de texto: un instrumento para su análisis. Avances de Investigación en Educación Matemática, 8, 73 - 94.

(C) Sociedad Española de Investigación en Educación Matemática (SEIEM). www.seiem.es 
group. We show examples of the mentioned research about how the instrument for analysis allows us to analyse the different kinds of problems that appear in textbooks.

Key words. Problem solving; mathematics textbooks; instrument for analysis.

\section{La résolution des problèmes dans les manuels: un instrument d'analyse}

\section{Résumé}

Cet article montre un instrument pour analyser le rôle de la résolution de problèmes dans les manuels scolaires. Nous commençons par la description du rôle donné aux manuels scolaires dans l'enseignement et l' apprentissage des mathématiques, puis passer à montrer un peu de recherche de l'enseignement des mathématiques, ont été menées sur les manuels scolaires, y compris ceux effectués placer dans notre groupe. Parmi ces exemples montrent comment l'outil d'analyse a pour analyser les types de problèmes qui apparaissent dans les manuels scolaires.

Paroles clés. Dépannage; manuels de mathématiques; outil d'analyse.

\section{Introducción}

Desde la aparición de la imprenta, la difusión de la cultura se ha materializado principalmente a través de los libros, que han facilitado el intercambio y la transmisión de ideas y saberes. La institución escolar también ha organizado su labor educativa alrededor de la tecnología impresa. Así, se ha creado un tipo especial y específico de material para que la institución escolar pueda funcionar: el libro de texto (Güemes, 1994), que se ha convertido en mediador entre las directrices curriculares y el trabajo del profesor en el aula (Villella y Contreras, 2005) y soporte básico del trabajo del alumnado (TIMSS, 2011).

El libro de texto es un recurso material utilizado habitualmente para organizar el trabajo en el aula, en el que se apoyan los estudiantes y profesores como guía en el proceso de enseñanza-aprendizaje (Martínez Bonafé, 2008; González y Sierra, 2004). Como cualquier otro material educativo, el libro de texto condiciona el tipo de enseñanza, máxime si el profesorado lo elige de manera exclusiva (Parcerisa, 1996), llegando a determinar el currículo más que la propia ley (Monterrubio y Ortega, 2012) y siendo a veces responsable de la trasmisión de errores e inconsistencias (Jaime, Chapa y Gutiérrez, 1992).

Las editoriales se convierten, así, en responsables de la traslación de lo relevante y de las formas de trabajo al aula, lo que conlleva una traslación al alumno y al profesor de las creencias del autor sobre las matemáticas y sus procesos de enseñanza y aprendizaje, sobre las formas de representación, sobre los procesos de demostración y prueba, sobre lo que es un problema, sobre el papel de la resolución de problemas (en adelante RP) en el aula, sobre la legitimidad de los contenidos que deben desarrollarse en el aula y que la sociedad valida como los necesarios para aprender (Olson, 1989), o sobre el rol que en su implementación refleja la actividad del docente, en muchos casos buscando en el libro la legitimidad de su proceder (Luke, Castell y Luke, 1989). Sin que siempre se sea totalmente consciente de ello, para muchos docentes la elección de un libro de texto supone de hecho la decisión curricular más importante, por lo que no es raro que este instrumento ejerza un efecto poderoso sobre sus enfoques de enseñanza y sobre las estrategias de aprendizaje de los alumnos (Villella y Contreras, 2005). 
Es esa consciencia de la relevancia del papel de los libros de textos la que ha motivado las investigaciones de nuestro grupo en este ámbito (Herdeiro, 2010; Guerrero, Carrillo y Contreras, 2014 y López y Contreras, 2014). En este artículo nos centraremos en el proceso que nos ha llevado a la elaboración de un instrumento de análisis del papel de la RP en los libros de texto de Educación Secundaria. En el apartado siguiente mostraremos algunas investigaciones que ponen de relieve la necesidad de investigar sobre los libros de texto, continuaremos mostrando los fundamentos de nuestro trabajo, en relación con lo que es para nosotros un problema matemático y sobre la tipología de problemas que se describe en la literatura, y terminaremos con la descripción del sistema de categorías que hemos elaborado para poder analizar los problemas que aparecen en los libros de texto. Para ayudar a comprender las diferencias entre las subcategorías, hemos usado los ejemplos de dos libros de texto ${ }^{i}$ que hemos analizado en nuestros últimos trabajos (Guerrero, Carrillo y Contreras, 2014; López y Contreras, 2014), a los que en adelante nos referiremos como Vincens Vives y Anaya.

\section{Relevancia de las investigaciones sobre libros de texto}

Dada la creciente importancia de los libros de texto en el ámbito educativo, éstos vienen siendo objeto de estudio desde hace varios años. Existen diversas investigaciones que analizan este tema desde perspectivas y fines diversos, como el análisis del uso de los materiales curriculares, los aspectos relativos al lenguaje y la legibilidad de los textos, la forma de presentación de los contenidos, el grado de desarrollo de los objetivos y contenidos curriculares en los manuales, etc. (Pino y Blanco, 2008; González y Sierra, 2004; Güemes, 1994); algunas han abordado el análisis historiográfico.

Además, se pueden encontrar numerosas investigaciones que se centran en el uso del libro de texto en el aula. Güemes (1994) examina el uso que dan los profesores a los libros de texto en la planificación y en el trabajo académico de aula, con el fin de relacionar las distintas prácticas docentes de uso del manual escolar con las creencias, concepciones o ideología del profesorado. Se concluye que el profesor debe desterrar la creencia de que el currículo se reduce al aprendizaje de unos conocimientos recogidos en un manual, para convertirse en un profesional reflexivo y crítico capaz de utilizar distintas herramientas de trabajo. Por otra parte, McNaught, Tarr y Sears (2010) analizan cómo usan los profesores el libro de texto a través de tres indicadores que permiten obtener una información mucho más sólida. Uno de estos indicadores representa el porcentaje del contenido del libro que los estudiantes han tenido oportunidad de aprender, mientras que los otros dos señalan la medida en que el libro de texto se usa en la enseñanza de contenidos de matemáticas.

Pino y Blanco (2008) estudian cómo reflejan los libros de texto las propuestas curriculares sobre la RP, centrándose en los contenidos de proporcionalidad y analizando ocho libros de texto de amplia difusión en España y Chile. Herdeiro (2010) examina el papel de la RP en los manuales escolares del último año de escolaridad obligatoria de Portugal. Trabaja tres temas distintos: Probabilidad y Estadística, Proporcionalidad inversa y Trigonometría del triángulo rectángulo, y escoge dos libros de texto, uno de ellos es el más usado en las escuelas del Algarve y el otro es el que usa la profesora en sus clases. Por último, Serrano (2012) analiza el papel de la RP en los textos de álgebra lineal para ingenieros, seleccionando seis libros a los que la autora recurre con frecuencia para la elección de actividades de aula. 
La selección y uso de libros de texto también ha sido objeto de investigación. Villella y Contreras (2005) mostraron cómo las concepciones de los profesores sobre los procesos de enseñanza y aprendizaje de las matemáticas condicionan la elección de criterios para elegir un libro y su uso en el aula.

La preocupación por la definición de criterios y la construcción de instrumentos que permitan evaluar la comprensión de conceptos y desarrollos presentados en un texto es más reciente. Un análisis de los libros de texto supone hacer hincapié en la estructura profunda de los mismos. Ésta está compuesta por la organización textual, trama conceptual y orientaciones para la construcción de significados (Rinaudo y Galvalisi, 2002).

Pareciera que un buen libro instructivo debe informar y explicar. Este requisito es puesto por Slater y Graves (1991) y atiende a la selección y presentación de los contenidos dentro del texto. De este modo, un libro instructivo no sólo debería informar al lector sobre un contenido particular sino que, además debería proporcionar explicaciones, mostrando con claridad las relaciones entre hechos, conceptos, teorías y contextos de observación u ocurrencia.

Se han elaborado varios instrumentos para el análisis y la evaluación de los libros de texto, con el fin de facilitar al profesorado la tarea de selección o valoración de los mismos. Entre ellos, destaca el modelo para la valoración de textos escolares de matemáticas creado por Monterrubio y Ortega (2012). En particular, existe una línea de investigación que ha explorado el papel de los problemas o de la RP en los libros de texto de matemáticas, sirviéndose de los anteriormente citados instrumentos de análisis. Además, en los últimos años estas investigaciones se han restringido a contenidos y niveles educativos específicos. El presente trabajo se inscribe en esta línea de investigación. En lo que sigue expondremos el proceso seguido en nuestra investigación para elaborar un instrumento para el análisis del papel de la RP en los libros de texto.

\section{Un marco para analizar el papel de los problemas en los libros de texto}

El instrumento para el análisis del papel de la RP en los libros de texto que constituye el núcleo de este apartado se ha construido partiendo de una manera determinada de concebir qué es problema y cuál es su papel en el currículo. Comenzamos exponiendo qué entendemos por problemas matemáticos, sus tipologías y el papel que ocupan en los diseños curriculares.

\section{1 ¿Qué entendemos por problema matemático?}

Diversos autores han otorgado distintos significados a problema matemático. A pesar de ello, todas las definiciones de problema tienen en común su consideración como una situación desconocida que requiere deliberación, imaginación y formulación de estrategias para llegar a su solución; la dificultad y el reto van ligados al concepto de problema.

Para Pólya (1962), "tener un problema significa buscar de forma consciente una acción apropiada para lograr un objetivo claramente concebido pero no alcanzable de forma inmediata” (p. 117). Para Krulik y Rudnik (1980) es una situación que necesita de una solución para la cual los resolutores no conocen medios o caminos evidentes para obtenerla. La idea de dificultad se manifiesta también en la siguiente 
definición: "una situación en la que se pide a un individuo realizar una tarea para la que no tiene un algoritmo fácilmente accesible que determine completamente el método de solución" (Charles y Lester, 1982, p. 5). Podría decirse entonces que un problema es una situación que produce un cierto grado de incertidumbre y que genera una conducta encaminada hacia la búsqueda de una solución (Pino, 2012).

Algunos autores destacan el carácter subjetivo de los problemas e insisten en que estas dificultades no son características intrínsecas de la situación, sino que dependen también del individuo que la aborda y del momento en que se aborda: "Lo que es un problema para una persona puede no serlo para otra, y lo que es un problema para una persona un día puede no serlo un próximo día" (Agre, 1982, p. 130). Para Schoenfeld (1985) "una tarea que requiere importantes esfuerzos para algunos estudiantes podría ser un ejercicio rutinario para otros (...) existe una relación particular entre el individuo y la tarea que hace de la tarea un problema para esa persona" (p. 74).

Carrillo (1998, p. 87) resalta la aplicación no rutinaria de conocimientos matemáticos en el proceso de resolución de un problema: "el concepto de problema debe asociarse a la aplicación significativa (no mecánica) del conocimiento matemático a situaciones no familiares, la consciencia de tal situación, la existencia de dificultad a la hora de enfrentarse a ella y la posibilidad de ser resuelta aplicando dicho conocimiento".

En definitiva, un problema es una situación en la que se hace necesario superar ciertos obstáculos para alcanzar los fines perseguidos y cuya consecución depende en gran medida de los conocimientos previos y el grado de compromiso del individuo que lo afronta.

Este carácter polisémico de problema se evidencia también en su uso en el aula. En el ámbito escolar, aunque los términos problema matemático o RP conviven diariamente entre los profesores de matemáticas. "nos será fácil encontrar a dos profesores que nos aporten, en esencia, una misma definición del término; un poco menos fácil que le otorguen el mismo papel en el currículo y bastante difícil que, de hecho, utilicen de igual forma la resolución de problemas en sus aulas" (Contreras, 1999, p. 51).

\subsection{Tipologías de problemas}

Los diferentes usos que los profesores dan a la RP en el aula pueden identificarse con los distintos tipos de problemas existentes. En la literatura podemos encontrar variadas tipologías de problemas, atendiendo a los criterios de clasificación utilizados.

Pólya (1985) establece una de las primeras clasificaciones de problemas, en la que distingue sólo dos tipos: el problema por resolver, cuyo propósito es descubrir la incógnita del problema, y el problema por demostrar, que consiste en mostrar de modo concluyente la exactitud o falsedad de una afirmación claramente enunciada. A partir de aquí se han formulado diferentes clasificaciones. Este trabajo toma como base las propuestas por Borasi (1986), Abrantes (1989) y Boavida (1993), ya que consideramos que son las más completas y explícitas. Borasi (1986), debido a su interés por mejorar la enseñanza a partir de la RP, intenta clarificar la noción de problema basándose en cuatro elementos estructurales: el contexto, o situación en la que se enmarca el problema; la formulación, o la forma en la que se presenta la tarea al alumno; la solución, o conjunto de soluciones del problema y el método de resolución, o el 
camino tomado para encontrar la solución. Sobre la base de estos cuatro elementos, la autora analiza lo que designa por: ejercicios, problemas de palabras, pruebas de una conjetura, problemas-enigmas, problemas de la vida real, situaciones problemáticas y situaciones.

Más tarde, Abrantes (1989) aplica los cuatro elementos estructurales definidos por Borasi para clasificar algunos ejemplos que considera aclaratorios. Este autor establece la siguiente clasificación: ejercicios, problemas de palabras, problemas para ecuacionar, problemas para demostrar, problemas para descubrir, problemas de la vida real, situaciones problemáticas y situaciones. Boavida (1993) realiza una síntesis de las clasificaciones de Borasi y Abrantes, para clasificarlos en: ejercicios, problemas de palabras, problemas para ecuacionar, problemas para demostrar, pruebas de una conjetura, enigmas/problemas para descubrir, problemas de la vida real, situación problemática y situación.

Esta última clasificación nos servirá de base para desarrollar el sistema de categorías de los tipos de problemas que hemos usado en nuestras investigaciones.

\section{Cómo hemos investigado el papel de la resolución de problemas en los libros de texto}

Las categorías que hemos utilizado en nuestras investigaciones para el análisis de los libros de texto son: tipos de problemas, contexto, formulación, tarea matemática y solución. A continuación, exponemos el proceso seguido para su elaboración, así como sus correspondientes subcategorías, de las que ofrecemos ejemplos extraídos de los temas de Geometría plana y Sistemas de ecuaciones lineales de los textos que hemos analizado (Guerrero, Carrillo y Contreras, 2014; López y Contreras, 2014), a los que, como hemos mencionado, nos referiremos como Vicens-Vives y Anaya, editoriales elegidas por ser las de mayor implantación durante el año 2011.

Debemos señalar que hemos dejado sin ejemplificar aquellas subcategorías para las cuales no se ha encontrado ningún ejemplo en los temas y libros mencionados.

\subsection{Tipos de problemas}

Como se mencionó anteriormente, partimos de la clasificación de Boavida (1993) para crear nuestra categoría tipos de problemas, aunque, dado que los problemas para ecuacionar de Abrantes (1989) pueden considerarse un caso particular de los problemas de palabras, en los que la traducción al lenguaje matemático se hacía a través de una ecuación, y las pruebas de una conjetura de Borasi (1986) podían considerarse un caso particular de los problemas para demostrar, estos dos tipos no fueron considerados. Asimismo, la situación problemática y situación que menciona Boavida (1993) se unieron en lo que llamamos problemas de la práctica matemática.

Así, inicialmente, en esta categoría se considera la subcategoría ejercicios, que, aunque realmente no cumplen la definición de problema, los consideramos, siguiendo la tradición de los autores mencionados, como problemas en los que basta reconocer o recordar un concepto específico o una definición, o aplicar un proceso algorítmico conocido para determinar la solución, son rutinarios y no requieren de la originalidad del resolutor, tal y como podemos apreciar en el problema 1: 
15. Resuelve los siguientes sistemas utilizando el método de sustitución:
a) $\left\{\begin{array}{l}x+2 y=18 \\ y-x=-9\end{array}\right.$
c) $\left\{\begin{array}{l}x+y=18 \\ y-x=4\end{array}\right.$
b) $\left\{\begin{array}{l}2 x+4 y=14 \\ x-2 y=-1\end{array}\right.$
d) $\left\{\begin{array}{l}x-y=8 \\ 2 y-x=-11\end{array}\right.$

Problema 1 (Vicens Vives, p. 83)

En este problema la única tarea a realizar consiste en conocer el método de sustitución para resolver sistemas de ecuaciones y aplicarlo en cada uno de los apartados.

La segunda subcategoría es la de problemas de palabras, caracterizados por estar enunciados en un contexto concreto que necesita traducirse al lenguaje matemático para su resolución, en los que toda la información necesaria para resolverlos aparece en el enunciado y, además, suele indicarse la estrategia a seguir. Al hilo del ejemplo anterior, podemos ver cómo el problema 2 bien podría resolverse con el mismo método de sustitución que nos pedía el problema 1, pero en este caso la resolución de dicho sistema aparece "camuflada" tras un enunciado contextualizado de manera más o menos cercana al alumno y cuya traducción es necesaria para resolver el problema.

$15 \nabla \nabla \nabla$ Por dos bolígrafos y tres cuadernos he pagado $7,80 €$; por cinco bolígrafos y cuatro cuadernos, pagué $13,20 €$. ¿Cuál es el precio de un bolígrafo? ¿Y de un cuaderno?

Problema 2 (Anaya, p. 122)

Los ejercicios y los problemas de palabras suponen, en nuestros estudios (Guerrero, Carrillo y Contreras, 2014; López y Contreras, 2014), el grueso de los problemas analizados, siendo las categorías que se nombran a continuación las menos comunes, llegando a ni siquiera aparecer reflejadas en algunas de las unidades.

En tercer lugar, consideramos los problemas para demostrar, como el problema 3: 


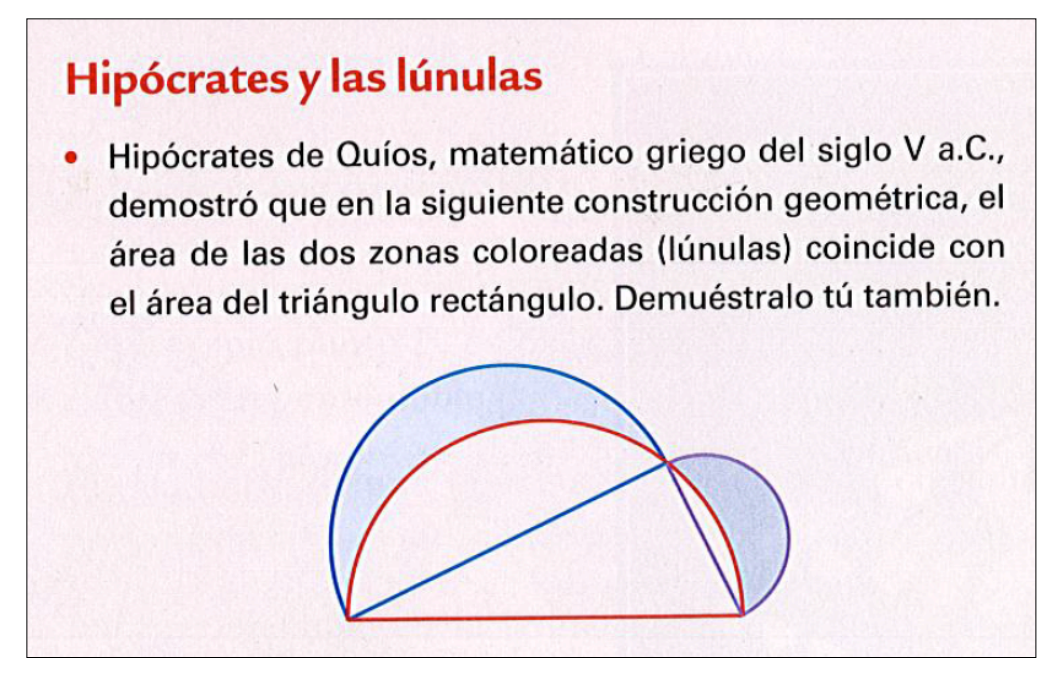

Problema 3 (Vicens Vives, p. 137)

El problema 3 solicita justificar la validez de la proposición, para cuya resolución se necesita recurrir a teoremas o propiedades relacionadas con la demostración solicitada y en los que se precisa del razonamiento deductivo, característica principal de esta subcategoría.

A continuación, consideramos los problemas para descubrir. Estos problemas suelen aparecer al final de cada unidad o con el nombre de enigma o desafío; en su formulación se pretende mostrar una forma atrayente, divertida o entretenida de aprender matemáticas y además las soluciones requieren lógica e ingenio. Un ejemplo de este tipo de problemas es el siguiente:

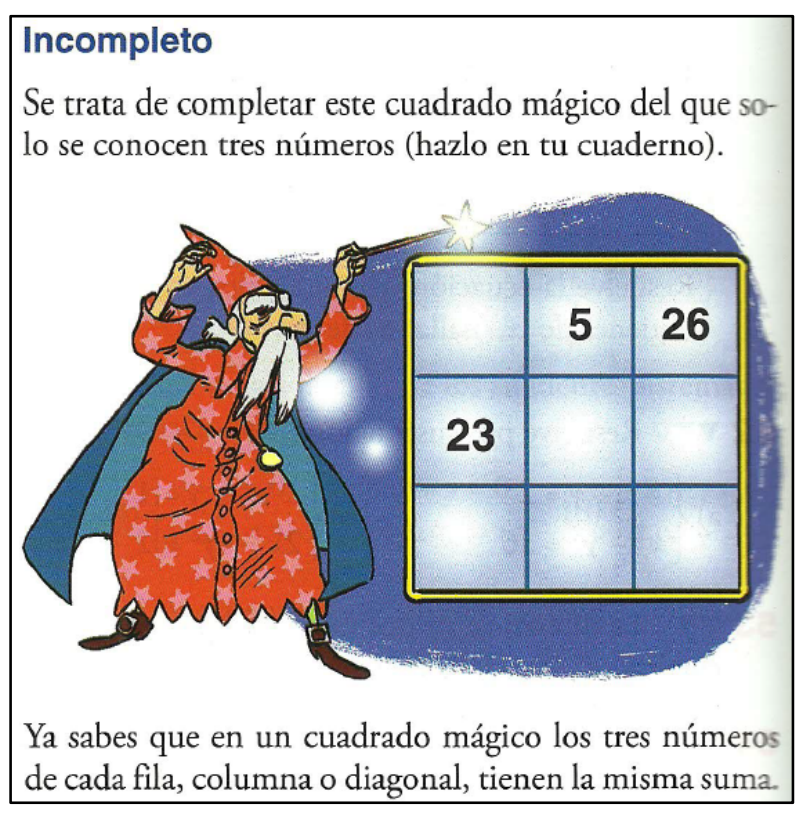

Problema 4 (Anaya, p. 126)

Los problemas de la vida real hacen referencia a situaciones factibles de darse en la vida real y que precisan de la construcción de diagramas, realización de estimaciones, cálculo de medidas o elaboración de análisis y síntesis. Permiten conocer 
las aplicaciones de las matemáticas en el mundo real y a veces no tienen solución exacta ni única.

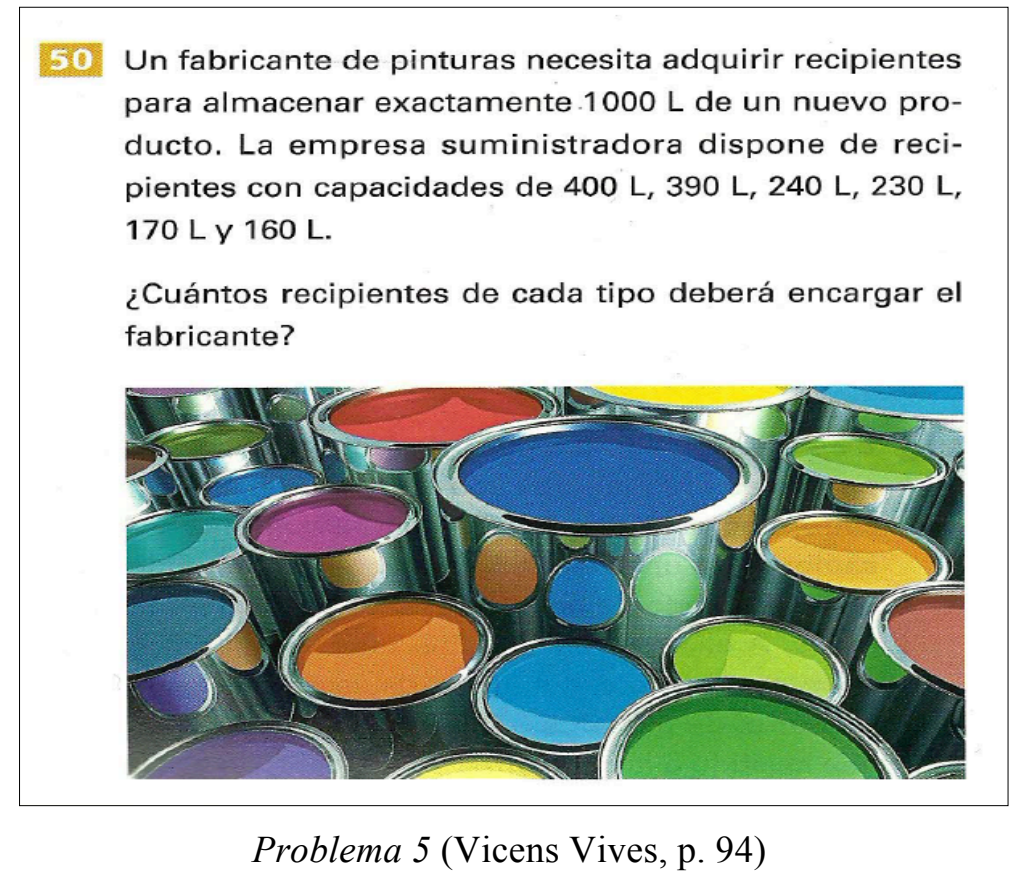

En la situación realista planteada en el problema 5 no se indica el método o algoritmo a seguir, sino que puede abordarse de varias maneras, como suele ocurrir en la vida real.

Por último, definimos una subcategoría denominada problemas de aplicación, la cual recoge un tipo específico de problemas de geometría plana, aquéllos que no encajan bien con la definición de problemas de palabras, porque no requieren de la traducción al lenguaje matemático como tal, pero que tampoco pueden considerarse ejercicios, puesto que plantean una situación en la que el alumno debe identificar un resultado relevante para encontrar la solución.

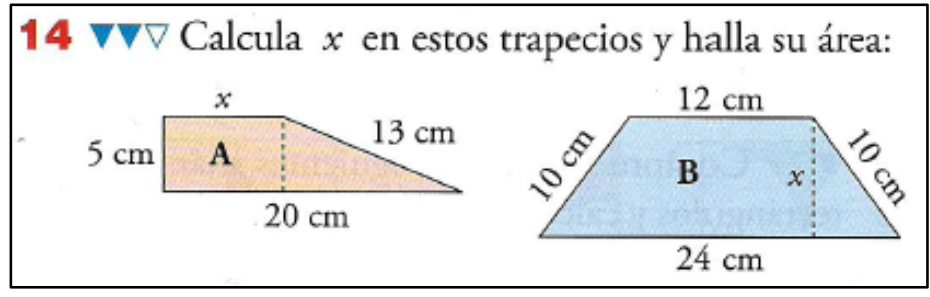

Problema 6 (Anaya, p. 183)

El problema 6 encaja en esta subcategoría puesto que su demanda cognitiva ${ }^{\text {ii }}$ asociada es mayor que la de los ejercicios, ya que se trata de una situación en la que es preciso identificar un resultado relevante con cuya aplicación se puede alcanzar la solución.

Además, añadimos los problemas de la práctica matemática, que recogen situación problemática y situación de Boavida (1993), pues su abordaje responde al tipo de acciones descritas a continuación. Los problemas de la práctica matemática permiten desarrollar procesos de exploración, formulación de hipótesis y su posterior validación, en los que se realizan conjeturas, verificaciones y argumentaciones ${ }^{\mathrm{iii}}$. El 
problema 7 es un ejemplo de esta subcategoría. Para resolver este problema, el alumno deberá explorar métodos y formular hipótesis de cómo podría resolverlo (cómo escoger los triángulos para hacer semejanza de triángulos y aplicar el teorema de Pitágoras de manera que se consiga averiguar la longitud AM). Finalmente, dado que todo lo que ha realizado serían hipótesis, debería validar su solución.

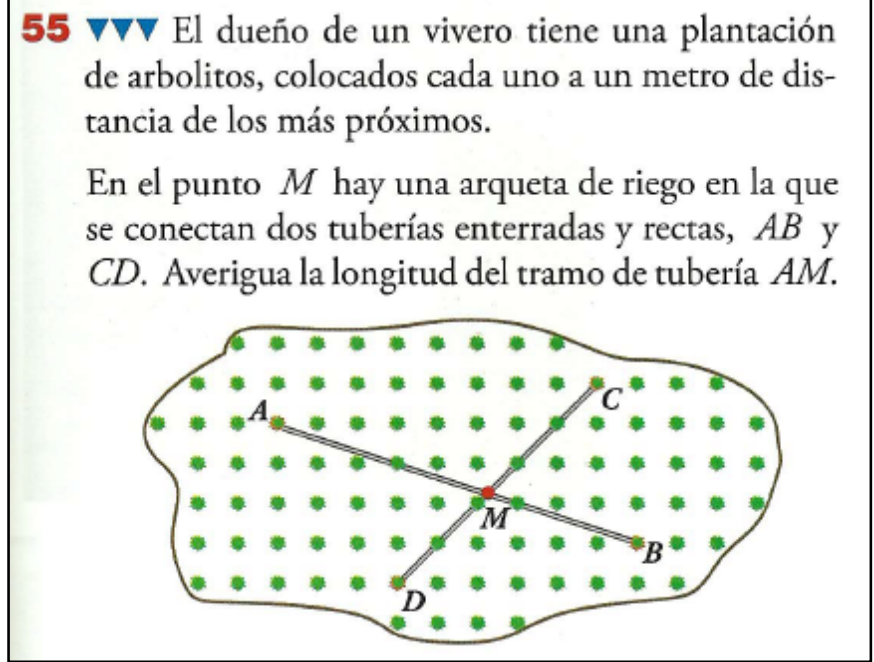

Problema 7 (Anaya, p.187)

\subsection{Contexto}

Inspirados fundamentalmente en Herdeiro (2010) y tomando algunas ideas de INECSE (2005) y Monterrubio y Ortega (2012), esta categoría incluye la contextualización en la realidad, contexto de datos proporcionados o contexto de conexión.

Herdeiro (2010) consideró que un problema podía estar explícitamente relacionado con el mundo real o con una representación plausible del mismo, o no estarlo, definiendo así las subcategorías contexto de la vida real y sin contexto de la vida real. Nosotros mantuvimos esta idea, pero nos pareció interesante volver a subdividir el contexto de la vida real en otras variantes, lo que aportaría mayor información acerca del contexto en el que se inserta el problema. De esta manera, tomando estas ideas de INECSE (2005), distinguimos entre contexto personal (problemas relacionados con actividades cotidianas), como el problema 2; laboral o educativo (situaciones que pueden darse en el centro escolar o algún entorno de trabajo), ejemplos de ello son los problemas 5 y 7; social (contexto relacionado con el entorno social y/o político en que se vive) y científico (problemas enmarcados en las ciencias naturales). Además, denotamos contexto puramente matemático al que Herdeiro (2010) nombra sin contexto de la vida real, entre los que podríamos enmarcar los problemas 1, 3, 4 ó 6.

En cuanto a los datos proporcionados, Herdeiro (2010) distingue entre contexto de datos genuinos o no genuinos. Nosotros los denotamos contexto de datos realistas (plausibles) o datos no realistas.

$6 \nabla \nabla \nabla$ Una fotografía de $15 \mathrm{~cm}$ de ancho y $10 \mathrm{~cm}$ de alto tiene alrededor un marco de $2 \mathrm{~cm}$ de ancho. ¿Son semejantes los rectángulos interior y exterior del marco? Responde razonadamente.

Problema 8 (Anaya, p. 182) 
El problema 8 tiene un contexto de datos realistas, ya que las medidas de la citada fotografía son medidas reales.

Por otro lado, Herdeiro (2010) considera el contexto con conexión con otras ramas de las matemáticas, con otras áreas disciplinares y sin conexión. Nosotros añadimos a esto el contexto con conexión con la historia de las matemáticas, extrayendo esta idea de Monterrubio y Ortega (2012).

Aunque la mayor parte de los problemas estudiados en nuestros trabajos (Guerrero, Carrillo y Contreras, 2014 y López y Contreras, 2014) son problemas sin conexión, encontramos algunos problemas con conexión con otras ramas de las matemáticas como el problema 9 (este problema se encuentra en la unidad de sistemas de ecuaciones y, sin embargo, su resolución involucra conceptos de geometría). Asimismo, el problema 3 es un ejemplo de problema con conexión con la historia de las matemáticas (las lúnulas de Hipócrates), siendo, junto con el problema 10, uno de los pocos que hemos encontrado en nuestro análisis dentro de esta clasificación.

$56 \nabla \nabla \nabla$ Las diagonales de un rombo se diferencian en $6 \mathrm{~cm}$ y su área es $56 \mathrm{~cm}^{2}$. Calcula la medida de las diagonales.

Problema 9 (Anaya, p. 125)

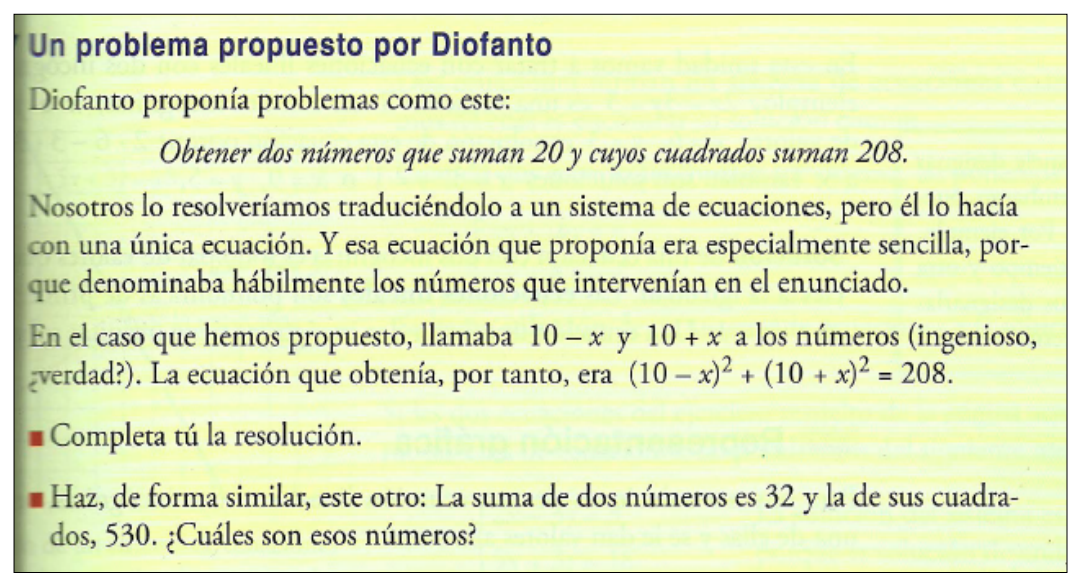

Problema 10 (Anaya, p. 111)

\subsection{Formulación}

De nuevo, sobre la base del trabajo de Herdeiro (2010), esta categoría engloba la ilustración, el número de cuestiones que presenta el problema desde el punto de vista sintáctico o semántico, las representaciones y los recursos empleados.

Al referirse a la ilustración, Herdeiro (2010) diferencia entre ilustración decorativa, representativa, informativa o sin ilustración. A todo esto nosotros sumamos la ilustración motivadora, que vemos como un caso intermedio entre la decorativa y la representativa. Así, finalmente consideramos ilustración decorativa (sin ninguna finalidad relacionada claramente con el problema), como la que aparece en el problema 5, ya que la imagen de las latas de pintura es simplemente un adorno 
del problema; motivadora (posible ayuda para el alumno pero que no aporta datos claramente significativos), siendo ejemplo de ello el problema 11 que se muestra a continuación, puesto que en la ilustración se puede apreciar que se añade una línea recta al dibujo, que puede llevar al estudiante a pensar en la posibilidad de dibujar un triángulo rectángulo que le ayude a resolver el problema, pero tal y como marca la definición no es una ayuda totalmente explícita, puesto que no se dibuja el triángulo en cuestión ni se colocan las medidas; representativa (aparecen datos que se dan en el enunciado), tal y como se puede ver en el problema 12; informativa (aparecen datos que no se aportan en el enunciado), como en el problema 6; o sin ilustración, de lo que sería un ejemplo el problema de palabras 2 .

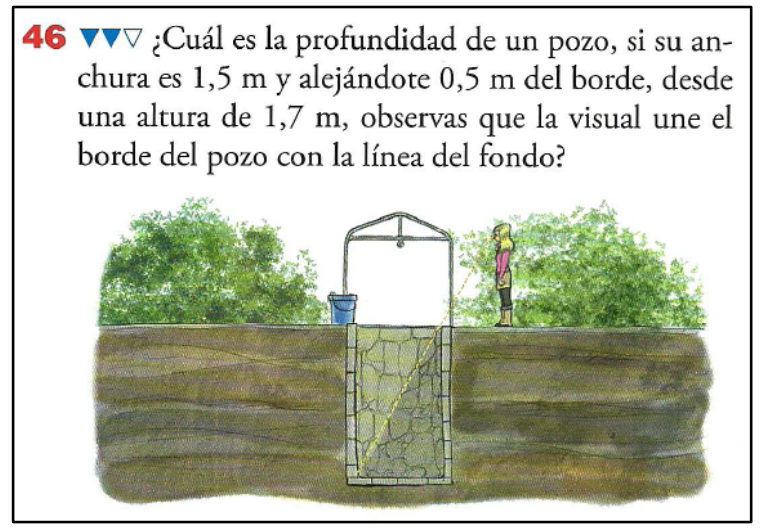

Problema 11 (Anaya, p. 186)

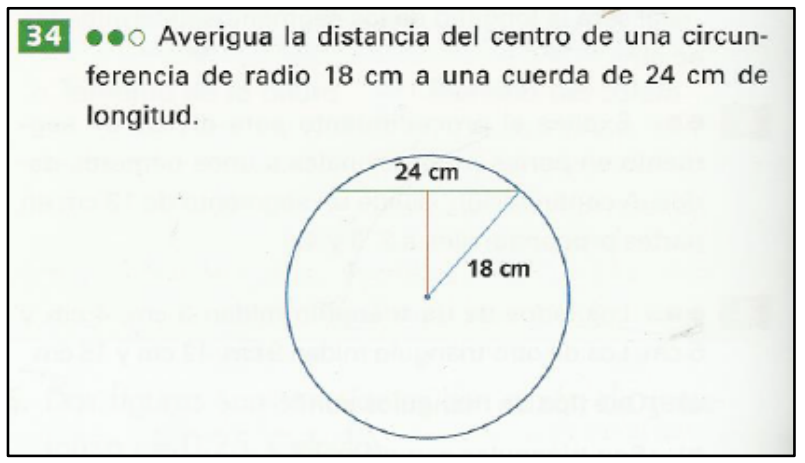

Problema 12 (Vicens Vives, p.134)

Herdeiro (2010) distingue entre formulación simple y agrupada según el número de cuestiones que presenta el problema desde el punto de vista sintáctico. Tras una primera aplicación del instrumento, nos pareció que los problemas también podían distinguirse según el número de cuestiones que planteaban desde el punto de vista semántico, así que añadimos esta nueva subcategoría a nuestro instrumento. De esta manera, tenemos formulación simple (una sola cuestión) y formulación agrupada (más de una cuestión en la misma actividad), según el número de cuestiones que presenta el problema desde el punto de vista sintáctico y formulación sencilla (una sola estrategia cognitiva) y formulación compleja (más de una estrategia cognitiva), según el número de cuestiones que presenta el problema desde el punto de vista semántico. Así, en un problema como el problema 1 podemos apreciar una formulación agrupada, pues aparecen cuatro cuestiones bajo un mismo enunciado, pero a su vez simple, pues desde 
el punto de vista semántico hay una sola cuestión, resolver cada sistema por el método de sustitución.

En cuanto a la información proporcionada en los problemas, tomamos la misma clasificación que Herdeiro (2010) en su trabajo, distinguiendo entre información suficiente (el enunciado del problema ofrece exactamente toda la información necesaria para la resolución del mismo), insuficiente (el enunciado del problema no ofrece toda la información necesaria para la resolución del mismo) y excesiva (el enunciado del problema ofrece toda la información necesaria para la su resolución y además añade información no relevante para resolverlo). Durante nuestro análisis pudimos observar que todos los problemas estudiados eran problemas con información suficiente para su resolución.

En su trabajo, Herdeiro (2010) analiza a qué tipo de recurso se recurre para representar los problemas, distinguiendo entre formulación exclusivamente verbal (problema 8), formulación con recurso de tabla (problema 4), con recurso de expresión algebraica (problema 1), utilizando una gráfica, referido a la gráfica de una función (problema 13), y con recurso diagrama (escala de probabilidades). Nosotros respetamos esta clasificación, y también añadimos la formulación verbal con ilustración (problema 6), además en la representación con diagrama no nos referimos a escala de probabilidades sino a cualquier tipo de diagrama aportado en el enunciando del problema, como diagramas de árbol o de barras. En nuestro análisis no encontramos ningún problema que utilice un diagrama en su formulación.

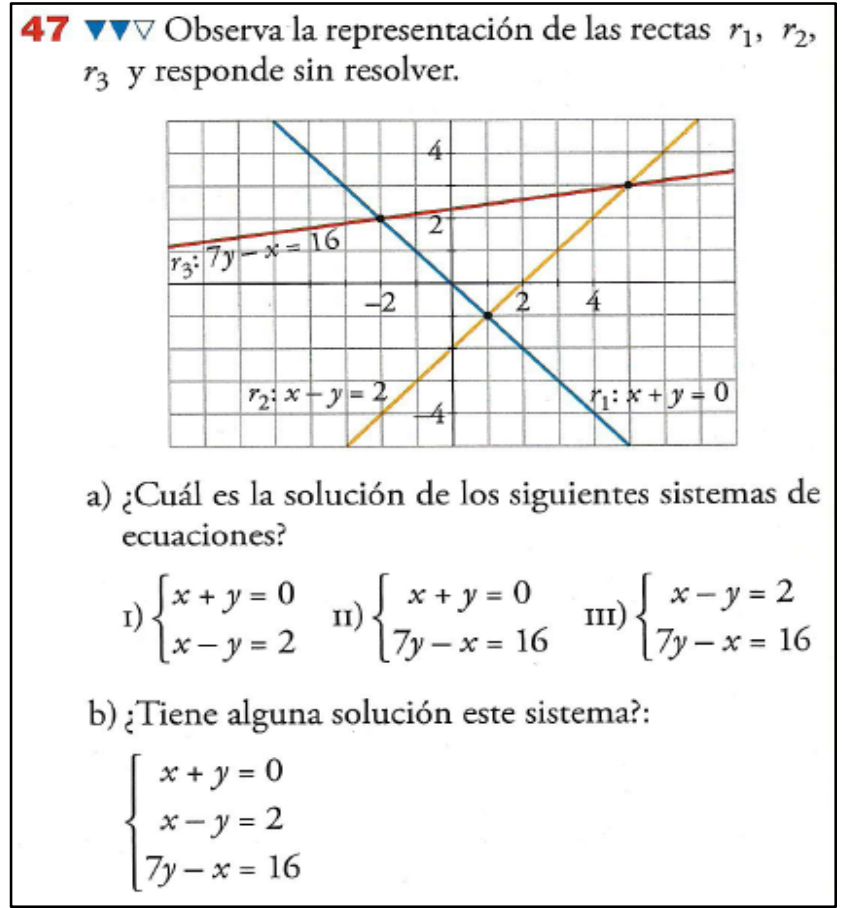

Problema 13 (Anaya, p. 124)

Con esto acabaría el análisis de la formulación realizado por Herdeiro (2010). En este apartado consideramos importante añadir una última subcategoría, pues creímos importante que en la formulación del problema se pudieran incluir aspectos como recursos recomendados para la realización del mismo con el objetivo de guiar o tratar de aportar algún elemento motivador a los estudiantes. Así distinguimos los recursos empleados, que pueden ser: materiales manipulativos, nuevas tecnologías o ningún recurso extra. 
En nuestro análisis no encontramos problemas que requiriesen del uso de nuevas tecnologías, pero sí de materiales manipulativos, como el problema 14.

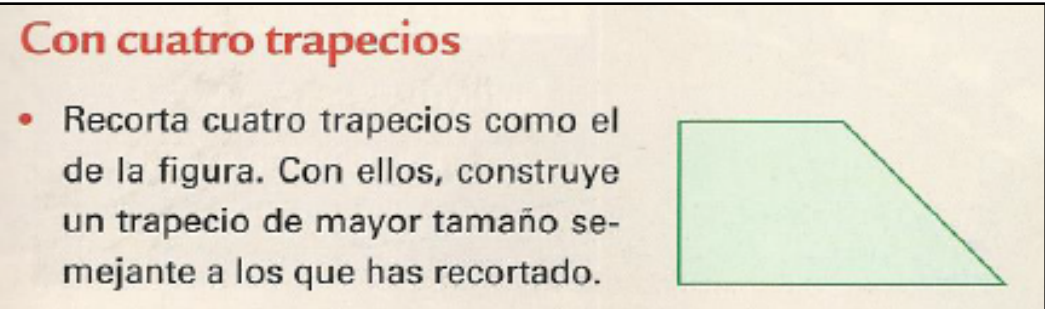

Problema 14 (Vicens Vives, p. 137)

\subsection{Tarea matemática}

Decidimos introducir la categoría Tarea matemática basándonos en los trabajos de Pino y Blanco (2008), e INECSE (2005). Esta categoría y la relativa de los tipos de problemas pueden verse como perspectivas complementarias; en una focalizamos la finalidad para la que se elabora el problema (tipos de problemas) y en la otra los requerimientos que le exigen al estudiante para su resolución (tarea matemática). Así en este apartado distinguiremos las subcategorías señaladas a continuación en relación con el mayor o menor grado de demanda cognitiva que exige el problema para ser resuelto, adecuado al nivel en el que se encuentran los estudiantes. Se puede ver que, en la propia definición de los tipos de problemas, aparece de manera implícita la tarea matemática. No obstante, decidimos hacer explícita la tarea matemática en sí misma, para observar sus aspectos de una manera más clara. Es por esta razón, por la que puede apreciarse un solapamiento entre estas dos categorías. Diferenciamos entre identificación y aplicación, razonamiento elemental o complejo e investigación.

La primera subcategoría, Identificación y aplicación, coincide con la idea de Pino y Blanco (2008), describiendo los problemas con un menor grado de exigencia, pues se trata de problemas familiares, que demandan básicamente la identificación y el empleo de conceptos sencillos y la aplicación de procedimientos rutinarios tales como los algoritmos. Estaríamos en el caso del problema 1, en el que tan sólo es necesario identificar y emplear el algoritmo del método de sustitución para resolverlo.

En cuanto a las dos siguientes subcategorías, en un principio pensamos en considerar una sola llamada razonamiento, en la que se podía encontrar cierto paralelismo con la categoría conexión, presente en INECSE (2005). Pero pronto vimos la necesidad de una modificación, puesto que buscábamos una mayor concreción del grado de exigencia cuando un problema demandase razonamiento. Distinguimos así entre Razonamiento elemental y Razonamiento complejo. La primera de ellas se asocia a problemas con un nivel mayor de exigencia que los de Identificación y aplicación, trascendiendo la mera repetición de algoritmos, conllevando su resolución la necesidad de razonamiento matemático y el establecimiento de relaciones entre distintas representaciones de una misma situación, o bien la conexión entre distintos aspectos, como ocurriría con el problema 2, en el que si bien hay que aplicar un método de resolución de sistema de ecuaciones no sólo basta con eso, sino que se requiere de un razonamiento matemático para plantear dicho sistema antes de iniciar su resolución. En cuanto al Razonamiento complejo, encontramos problemas en los que predomina el razonamiento matemático, que pueden considerarse un paso previo a los problemas de investigación, pues aunque requieren establecer relaciones más complejas que las anteriores, suelen tener una respuesta única y exacta y no requieren de generalización, o descubrimiento de regularidades o conjeturas ni justificación de los resultados, tal y 
como sucede en el caso del cuadrado mágico del problema 4. Por último (coincidiendo con Pino y Blanco, 2008), fijamos un mayor nivel de exigencia con la categoría Investigación, en la que se enmarcan problemas cuya resolución requiere cierta comprensión y reflexión por parte del estudiante, creatividad tanto para identificar conceptos como para enlazar conocimientos y procesos matemáticos, como ocurre para encontrar la demostración que se pide en el problema 3. Este tipo de problemas exigen investigación, descubrimiento, generalización, manipulación para descubrir regularidades o verificar conjeturas y explicación o justificación de los resultados. Puede tratarse de problemas abiertos o sin respuesta única.

\subsection{Solución}

La última categoría que introdujimos en nuestro instrumento de análisis es la Solución, formulada sobre la base de lo planteado por Herdeiro (2010). En esta categoría se hace referencia a la solución del problema, entendiendo la solución como resultado esperable, distinguiendo entre repuesta cerrada o abierta, representaciones pedidas, unicidad y exactitud y toma de decisión en cuanto a las soluciones.

Tras modificar ligeramente esta parte del trabajo de Herdeiro (2010), nuestro instrumento distingue en esta categoría entre respuesta cerrada y abierta, representaciones pedidas en las respuestas, unicidad y exactitud de la solución y necesidad de toma de decisión. Entendemos que cuando hablamos de respuesta cerrada y abierta no podemos englobar todas ellas en un mismo conjunto y que cada tipo aporta una información diferente sobre la exigencia del problema; los tipos de respuestas cerradas que contemplamos son corta, de desarrollo, de completitud, de tipo verdadero/falso, de asociación o correspondencia o de elección múltiple. En cuanto a la respuesta abierta distinguimos entre corta, de desarrollo o cualquier tipo de respuesta cerrada con respuesta abierta de desarrollo (unificando en esta última varias de las clasificaciones que contempla Herdeiro (2010) en este apartado).

El problema 9 sería un problema de respuesta cerrada corta, pues el enunciado sólo pide al resolutor dos números, en este caso dos medidas. El problema 8 sigue siendo de respuesta cerrada pues se debe responder sí o no a la semejanza de los rectángulos mencionados, sin embargo, en este caso es de desarrollo, pues la solución requiere de un razonamiento que hace que la respuesta no se reduzca a una mera palabra. En el problema 4 encontraríamos un ejemplo de respuesta cerrada de completitud, pues se trata de completar el cuadrado mágico con números. Las respuestas abiertas son las menos numerosas, pudiendo señalar el problema 15 como ejemplo de respuesta abierta corta, pues se trata de dar un solo sistema y éste queda abierto al resolutor.

$$
\begin{gathered}
42 \nabla \nabla \nabla \text { Escribe un sistema de dos ecuaciones con dos } \\
\text { incógnitas cuya única solución sea } x=2, y=-1 \text {. }
\end{gathered}
$$

Problema 15 (Anaya, p. 124)

Después de esto, Herdeiro (2010) diferencia entre respuestas con justificación y respuestas sin justificación, clasificación que decidimos eliminar tras considerar que cualquier problema matemático conlleva implícitamente una justificación.

Al referirnos a las representaciones pedidas añadimos a la clasificación de Herdeiro (2010) la representación numérica o verbal y eliminamos la representación 
con recurso de escala de probabilidades, pues, al igual que en la información aportada en la categoría de formulación, no nos referimos a escala de probabilidades sino a cualquier tipo de diagrama que se pida en la solución. De este modo, distinguimos entre representación exclusivamente numérica o verbal (como la que se da en el problema de palabras 2), utilizando una ilustración (problema 16 presentado a continuación), una gráfica (problema 17), un diagrama, una tabla (problema 18) o una expresión algebraica (problema 1).

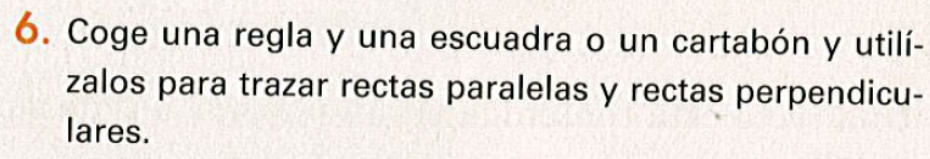

6. Coge una regla y una escuadra o un cartabón y utilizalos para trazar rectas paralelas y rectas perpendiculares.

Problema 16 (Vicens Vives, p. 118)

$$
\begin{aligned}
& 4 \nabla \nabla \nabla \text { a) Representa gráficamente en los mismos ejes } \\
& \text { las dos rectas siguientes: } \\
& \begin{array}{c}
2 x+y=3 \\
x-y=3
\end{array}
\end{aligned}
$$

Problema 17 (Anaya, p. 121)

$11 \bullet \circ \circ \mathrm{Haz}$ una tabla de valores para resolver el sistema:

$$
\left\{\begin{array}{l}
x+2 y=18 \\
3 x-4 y=4
\end{array}\right.
$$

Problema 18 (Vicens Vives, p. 91)

Al hablar de solución diferenciamos Unicidad y exactitud (distinguiendo entre solución única y exacta o solución no única ni exacta) y Toma de decisión (diferenciando entre resolución con o sin toma de decisión en cuanto a las soluciones). Por ejemplo, el problema 1 (expuesto más arriba) sería de solución única y exacta, pues sobra decir que la única solución posible es la solución de cada sistema, que es un resultado exacto, por ser estos compatibles determinados. En este caso no habría toma de decisión, pues ya se indica en el enunciado que se deben resolver los sistemas por el método de sustitución. En cambio, el problema 19, aunque también es de solución única y exacta (y coincide con el problema 1 en todas las subcategorías), sí que implica toma de decisión, pues es el estudiante quien debe decidir el método a emplear para resolver los sistemas. 


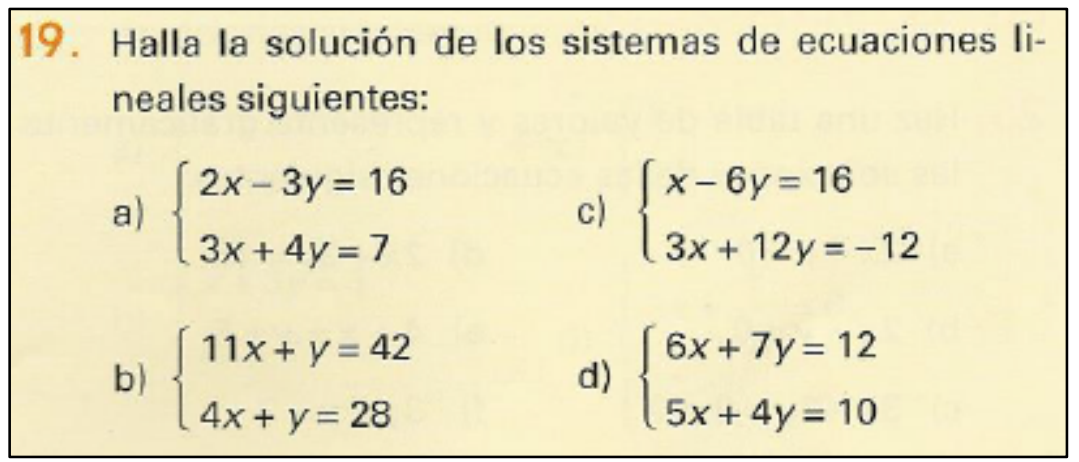

Problema 19 (Vicens Vives, p.85)

Por último, y como ejemplo de lo que sería un problema de respuesta no única ni exacta, tenemos el problema 20, en cual se pide dar ejemplos de objetos que puedan identificarse con un punto, una recta o un plano. La respuesta es no única ni exacta porque admite diferentes soluciones.

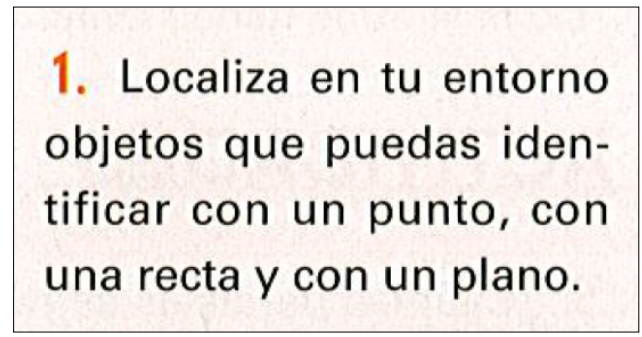

Problema 20 (Vicens Vives, p.117)

\section{Reflexiones finales}

Como se aprecia en el epígrafe anterior, el instrumento presentado ha mostrado variabilidad y versatilidad para clasificar los problemas de los libros de texto. Hemos sido sensibles a la aparición de posibles características o tipos de problemas, efectuando las incorporaciones necesarias al instrumento. Ahora bien, su utilidad, pensamos, trasciende el campo de la investigación. El equipo directivo de un centro, o el profesorado del Departamento de Matemáticas, tiene en este instrumento un compendio de criterios que puede emplear para seleccionar el libro de texto.

En cuanto al análisis específico de los textos Vicens Vives y Anaya, podemos concluir que no se promueve la RP como eje central del aprendizaje, puesto que la mayoría de los problemas propuestos son ejercicios, seguidos por problemas de palabras, siendo ambas las subcategorías de complejidad más baja. Llama la atención que el currículo de matemáticas de Educación Secundaria apueste por una enseñanza basada en la RP, que la investigación la contemple como parte integral de las matemáticas, pero que, sin embargo, la principal herramienta de trabajo en el aula no sea coherente con estas ideas, y que sólo aparezcan problemas en los que se pone en marcha la lógica, el ingenio o problemas cercanos a la vida real en lugares puntuales de la unidad. Así, parece que se transmite una idea de las matemáticas como herramienta de cálculo, basadas en el aprendizaje de fórmulas (que pueden olvidarse fácilmente) y que sólo ayudan a resolver ejercicios. 
Estudios futuros centrados en otros temas matemáticos y en otras editoriales pondrán a prueba el instrumento diseñado. En cualquier caso, no obstante, los resultados obtenidos, además de poner de relieve la utilidad metodológica del instrumento, aportan elementos de reflexión sobre la coherencia de los libros de texto (en relación, entre otras cosas, con las recomendaciones curriculares y las que provienen de la investigación en educación matemática), sobre la diversidad y variabilidad de las tareas a valorar en los libros, atendiendo a algunos de los criterios expuestos anteriormente, y sobre el uso que el profesorado haga de los mismos en la planificación y el desarrollo de su enseñanza.

\section{Referencias}

Abrantes, P. (1989). Um (bom) Problema (nao) é (só)...Educaçao e Matemática, 8, 710.

Agre, G.P. (1982). The Concept of Problem. Educational studies in mathematics, 13(2), 121-142.

Boavida, A.M. (1993). Resoluçao de problemas em educaçao matemática. Master's thesis. Universidade Nova de Lisboa.

Borasi, R. (1986). On the nature of problems. Educational Studies in Mathematics, $17(2), 125-141$.

Carrillo, J. (1998). Modos de resolver problemas y concepciones sobre la matemática y su enseñanza: metodología de investigación y relaciones. Huelva: Universidad de Huelva Publicaciones.

Carrillo, J., Climent, N., Contreras, L.C. y Muñoz-Catalán, M.C. (2013). Determining specialised knowledge for mathematics teaching. En B. Ubuz, C. Haser y M.A. Mariotti (Eds.), Proceedings of the ERME 8, (pp. 2985-2994). Middle East Technical Universitiy: Ankara, Turquía.

Charles, R. y Lester, F. (1982). Teaching Problem Solving: What, why and how. Palo Alto: Dale Seymour Publications.

Contreras, L.C. (1999). Concepciones de los Profesores sobre la Resolución de Problemas. Huelva: Servicio de Publicaciones de la Universidad de Huelva.

González, M.T. y Sierra, M. (2004). Metodología de análisis de libros de texto de matemáticas. Los puntos críticos en la enseñanza secundaria en España durante el siglo XX. Enseñanza de las Ciencias, 22(3), 389-408.

Güemes, R.M. (1994). Libros de texto y desarrollo del currículo en el aula. Un estudio de casos. Tesis Doctoral. Universidad de La Laguna.

Guerrero, A.C., Carrillo, J. y Contreras, L.C. (2014). Problemas de Sistemas de Ecuaciones Lineales en libros de texto de $3^{\circ}$ ESO. En M. T. González, M. Codes, D. Arnau y T. Ortega (Eds.), Investigación en Educación Matemática XVIII, (pp. 395-404). Salamanca: SEIEM.

Herdeiro, C. (2010). A resoluçao de problemas nos manuais escolares de matemática do $9^{\circ}$ ano de escolaridade. Tesis Doctoral. Universidad de Huelva.

INECSE (2005). PISA 2003: Pruebas de Matemáticas y de Solución de Problemas. Madrid: MEC.

Jaime, A., Chapa, F. y Gutiérrez, A. (1992). Definiciones de triángulos y cuadriláteros: errores e inconsistencias en libros de texto de E.G.B. Epsilon, 23,49-62. 
Krulik, S. y Rudnik, K. (1980). Problem solving in school mathematics. Year Book. Reston, VA: NCTM.

López E.M. y Contreras L.C. (2014). Análisis de los problemas matemáticos de un libro de texto de $3^{\circ} \mathrm{ESO}$ en relación con los contenidos de geometría plana. En M. T. González, M. Codes, D. Arnau y T. Ortega (Eds.), Investigación en Educación Matemática XVIII, (pp. 425-434). Salamanca: SEIEM.

Luke, C., Castell, S y Luke, A. (1989). Beyond criticism: the authority of the school textbook. En C. Luke, S. de Castell y A. Luke (Eds.), Language authority and criticism. Readings on the School Textbook. (pp. 123-146). London: Falmer Press

Martínez Bonafé, J. (2008). Los libros de texto como práctica discursiva. Revista de la Asociación de Sociología de la Educación 1(1), 62-73.

McNaught, M.D., Tarr, J.E. y Sears, R. (2010). Conceptualizing and Measuring Fidelity of Implementation of Secondary Mathematics Textbooks: Result of a Three-Year Study. Paper presented at the Annual Meeting of the American Educational Research Association. Denver, CO.

MEC (2012). Panorámica de la Edición Española de Libros 2011. Madrid: MEC.

Monterrubio, M.C. y Ortega, T. (2012). Creación y aplicación de un modelo de valoración de textos escolares matemáticos en Educación Secundaria. Revista de Educación, 358, 471-496.

Olson, D. (1989). On the language and authority of textbooks. En C. Luke, S. de Castell y A. Luke (Eds.), Language authority and criticism. Readings on the School Textbook (pp.86-98). London: Falmer Press.

Parcerisa, A. (1996). Materiales curriculares. Cómo elaborarlos, secuenciarlos y usarlos. Barcelona: Graó.

Pino, J.A. (2012). Concepciones y prácticas de los estudiantes de Pedagogía Media en Matemáticas con respecto a la Resolución de Problemas $y$, diseño $e$ implementación de un curso para enseñar a resolver problemas. Tesis Doctoral. Universidad de Extremadura.

Pino, J. y Blanco, L.J. (2008). Análisis de los problemas de los libros de texto de matemáticas para alumnos de 12 a 14 años de edad en España y Chile, en relación con los contenidos de proporcionalidad. Publicaciones, 38, 63-88.

Pólya, G. (1962). Mathematical discovery. On Understanding, Learning and Teaching Problem Solving. Nueva York: John Wiley \& Sons.

Pólya, G. (1985). Cómo plantear y resolver problemas. México: Trillas.

Rinaudo, M. y Galvalisi, C. (2002). Para leerte mejor... Cómo evaluar la calidad de los libros escolares. Buenos Aires: La Colmena.

Schoenfeld, A.H. (1985). Mathematical Problem Solving. Nueva York: Academic Press.

Serrano, I. (2012). Análisis de los problemas de libros de texto de Álgebra Lineal. Trabajo Fin de Máster. Universidad de Huelva.

Slater, W. y Graves, M. (1991). Investigaciones sobre el texto expositivo. En D. Muth (Ed.), El texto expositivo (pp.132-145). Buenos Aires: Aique.

Stein, M.K., y Smith, M.S. (1998). Mathematical Tasks as a Framework for Reflection: From Research to Practice. Mathematics Teaching in the Middle School, 3, 268-275. 
TIMSS (2011). TIMSS 2011 Assessment Framewoks. Boston: TIMSS \& PIRLS International Study Center Lynch School of Education, Boston College.

Villella, J. A. y Contreras, L.C. (2005). La selección y uso de libros de texto: un desafío para el profesional de la enseñanza de la matemática. La Gaceta de la RSME, 8(2), 419-433.

\section{Referencia de los autores}

Elena María López, Universidad de Huelva (España) elenalg@hotmail.com

Ana Cristina Guerrero, IES Licinio de la Fuente (España) acguerrerog@gmail.com

José Carrillo, Universidad de Huelva (España) carrillo@uhu.es

Luis Carlos Contreras, Universidad de Huelva (España) lcarlos@uhu.es 


\title{
Problem solving in textbooks: an instrument for analysis
}

\author{
Elena María López, Universidad de Huelva (España) elenalg@hotmail.com \\ Ana Cristina Guerrero, IES Licinio de la Fuente (España) acguerrerog@gmail.com \\ José Carrillo, Universidad de Huelva (España) carrillo@uhu.es \\ Luis Carlos Contreras, Universidad de Huelva (España) lcarlos@uhu.es
}

\begin{abstract}
The textbook plays an essential role in the learning-teaching process as well as being a vital tool in the classroom. This is one of the most used resources by teachers, becoming a basic guide for students and educators that even determines the way of teaching. This fact shows the importance for textbooks address the necessary issues and correctly in each subject. Furthermore, talking about mathematic education, problem solving has been one of the main focuses of research and discussion in the last 30 years. The ability to solve problems has become an essential content in the teaching and learning of mathematics in primary and secondary school curricula in different countries. The proposed curriculum for these levels considers problem solving as a central focus in Mathematics. This is the reason why there is a wide variety of researches about how problem solving is treated in textbooks and about how these manuals are selected and used by the instructors.

This awareness leads to investigate about the definition and construction of certain instruments in order to analyse the internal structure of textbooks. In particular, there is a line of research that explores the role of problem solving in math texts, using these kinds of analytical instruments and focusing on certain content and educational levels. In this line of work, we show here an instrument to analyse the role of problem solving in textbooks. Understanding that a math problem is an unfamiliar situation where it is necessary to overcome certain obstacles to achieve the pursued aims and that requires deliberation, imagination and strategies to reach a solution, we start by distinguishing seven different kinds of problems, basing our definitions in previous and notorious works. This first category is followed by other four ones, where we focus on the context in which the problems are set, the formulation associated to these, the mathematical task required to find the solution to the problem studied and finally we analyse the solution understanding this as the expected result of the problem. In each category and subcategory we show examples taken from the books that we have analysed in our last works. With these examples we try to get a better understanding of the instrument and verify its utility.

Finally, we conclude that this instrument is useful to classify the problems that appear in mathematical textbooks. What is more, teachers could find in it a useful tool to decide which textbooks to select. According to the specific research of the two textbooks we have analyzed in previous works, it is noteworthy that there are important features that are inconsistent which those that appear in the official curricula, as most of the problems analysed are exercises of concepts and algoritms applications which require a very low cognitive demand. Future studies could test this instrument; anyway, we think that it offers not only a methodological utility but also a reflection tool about the texts quality and the useful and election that teachers make of textbooks.
\end{abstract}


i Colera, J., Colera, L., Gaztelu, I. y Oliveira, M.J. (2011). Matemáticas 3 Educación Secundaria. Madrid: Anaya. Pancorbo, L. (2011). Matemáticas 3 Educación Secundaria. Nuevo Vector. Barcelona: Vicens Vives.

ii Se usa demanda cognitiva inspirados en Stein y Smith (1998), quienes diferencian cuatro niveles: memorización, procedimiento sin conexión o significado, procedimiento con conexión y significado y hacer matemáticas. Estos autores consideran la demanda cognitiva como una noción asociada al nivel de complejidad e implicación que la tarea requiere.

iii Usamos la expresión Práctica Matemática en el mismo sentido que lo hacen Carrillo, Climent, Contreras y Muñoz-Catalán (2013), refiriéndose, en ese caso, a un subdominio del conocimiento especializado del profesor de matemáticas. 